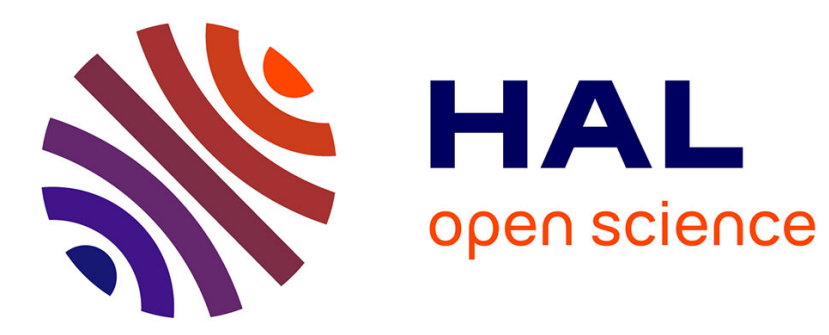

\title{
Des signes à la littérature: problèmes de langage dans l'Histoire comique de Francion
}

Emmanuel Desiles

\section{To cite this version:}

Emmanuel Desiles. Des signes à la littérature: problèmes de langage dans l'Histoire comique de Francion. Littératures classiques, 2001. hal-01857006

\section{HAL Id: hal-01857006 \\ https://hal.science/hal-01857006}

Submitted on 14 Aug 2018

HAL is a multi-disciplinary open access archive for the deposit and dissemination of scientific research documents, whether they are published or not. The documents may come from teaching and research institutions in France or abroad, or from public or private research centers.
L'archive ouverte pluridisciplinaire HAL, est destinée au dépôt et à la diffusion de documents scientifiques de niveau recherche, publiés ou non, émanant des établissements d'enseignement et de recherche français ou étrangers, des laboratoires publics ou privés. 


\section{Des signes à la littérature : problèmes de langage dans l'Histoire comique de Francion}

Dire du dire serait bien l'une des caractéristiques fédératrices de nombreux textes au début du XVIIème siècle. Rendre compte de la parole, alors même qu'on l'utilise, devient une habitude chez les écrivains du temps, et quelquefois une valorisation de leur production. Pour le poète Jean Godard, il existe deux langages distincts « Le premier, quelque abondant qu'il puisse être en parolles, deuiendroit pourtant muet : s'il falloit qu'il vînt à randre raison de son dire. Mais le second, capable de parler de toutes choses en bons termes, ne manque point de bons discours à soi-même : ains fait heureusemant tourner ses parolles en son honneur, \& parler de soi-même par sa bouche" ». Godard surenchérit plus loin, et définit le "vulgaire ignorant » celui « qui parle sans pouuoir randre raison de sa parolle ${ }^{2}$ ».

Incluse dans un corpus de romans comiques et satiriques eux-mêmes très enclins à développer ce genre d'analyses, l'Histoire comique de Francion s'en fait une nécessité. « Si l'on est curieux du langage » - « comme en effect l'on le doit estre » prend le soin de préciser Sorel - « où le peut on considerer mieux qu'icy ${ }^{3}$ » ? C'est assez inviter son lecteur à s'attacher à la perspective métalangagière du roman. L'histoire de Francion ne se borne donc pas au parcours du héros, mais s'ouvre sur l'univers polymorphe des langages - ceux du texte comme ceux du contexte culturel dans lequel il avait été créé. Car réduire la réflexion sorélienne aux seuls mots serait elliptique. L'auteur de Saint-Germain-l'Auxerrois considère bien des aspects du langage, des musiques, des danses, des peintures, jusqu'aux gestes. Tout ce qui peut représenter le monde - ou tente de la faire - intéresse Sorel. Et pourtant...

\section{I. « Je ne vous la sçaurois representer » (Francion, 1623 : livre VII) ${ }^{4}$}

Certes, le geste est signifiant et Sorel le reconnaît. Il en fait un trait plus spécifiquement italien : « il n'y avoit que mes yeux qui parloient », raconte Francion à propos de ses entrevues avec Emilie, « mais dans ces pays une simple œillade ou une petite action en disent souvent davantage que les plus longs entretiens des autres nations ${ }^{5} \gg$. Ce n'est d'ailleurs pas un hasard si c'est dans cette même Rome que Francion a l'idée d'une comédie fondée seulement sur des gestes et des signes ${ }^{6}$. Pouvoir ainsi structurer toute une pièce de théatre à partir de la gestuelle, rappelle non seulement une veine dramaturgique existante, celle des mimes, mais également le pouvoir de suggestion de cette même gestuelle. La pièce a en outre l'avantage d'être cosmopolite et de ramener le lecteur dans un temps antébabélique.

Toutefois, bien des situations du Francion rappellent que le geste ne suffit pas. La parole l'accompagne dans la majorité des cas. De Collinet qui parle «avec des gestes extravagants ${ }^{7} »$ ou $~ "$ avec des actions et des tournoiements d'yeux admirables ${ }^{8} »$, à Francion qui avoue chanter en faisant « des grimasses, des gestes et des postures, dont tous les bouffons

\footnotetext{
1 Jean Godard, La Langue Françoise, Lyon, 1620, Reprint Slatkine, Genève, 1972, p. 34.

2 Ibid., p. 37.

${ }^{3}$ Charles Sorel, Histoire comique de Francion, éd. Emile Roy, Hachette, Société des Textes Français Modernes, Paris, 1924-1931, tome III, p.125. Toutes nos références et citations renverront à cette édition, la seule encore à ce jour à proposer de façon complète les trois états du texte.

4 Ibid., tome III, p.22.

${ }^{5}$ Ibid., tome IV p.93.

6 « Francion les voulut contenter d'une autre façon, il joüa le lendemain une autre Comedie que toutes sortes de nations pouvoient entendre, car tout ne s'y faisoit que par signes ». Ibid., tome IV, p.63.

${ }^{7}$ Ibid., tome II., p. 139.

${ }^{8}$ Ibid., tome II, p. 151.
} 
de l'Europe seroient bien ayses d'avoir de la tablature pour en gaigner leur vie' ", le geste est un auxiliaire de la parole verbale demeurée souveraine dans l'acte de communication. D'ailleurs, il arrive que dans le roman l'utilisation unique du geste vaille pour rupture de communication. Quand la dame aimée de Bajamond ne répond à sa servante qui l'a trahie « que par un signe de la teste ${ }^{10}$ », quand Francion lui-même, chez Luce, se métamorphose en " une souche sans parole, qui ne respondit que par quelque signe de la teste" $"$, les personnages utilisent sciemment l'aspect réducteur du langage gestuel. La leçon avait déjà été formulée dans le songe du héros éponyme. Le vieillard du rêve « qui avoit de grandes oreilles et la bouche fermée d'un cadenas » est réduit au silence, ou plus exactement à la parole gestuelle. Francion précise : " il me monstra de la main un petit boccage, comme s'il m'eust voulu dire que c'estoit là que je pourrois avoir response de ce que je luy demandois ${ }^{12} \gg$. Autrement dit, le geste n'est qu'un renvoi à une autre parole, plus précise, et ne saurait suffire dans une entreprise communicative. Sorel a pris soin quelques pages auparavant de nous rappeler que les vicieux, monstres oniriques, ont précisément la caractéristique de ne pouvoir parler : «Ils me firent entendre par signes qu'ils me pardonneroient [...] car comme je vous ay desjà dit, ils n'avoient point de voix humaine ${ }^{13} \gg$. On voit bien ainsi comment Sorel répète sa leçon, réitère son enseignement, en confirmant que l'usage de la parole est le privilège - et pourquoi pas le propre - de l'homme. Bref, après avoir étudié le langage gestuel, Sorel en avait quelque peu épuisé les possibilités. La place demeurait libre pour les autres langages, à commencer par le verbe qui l'avait supplanté.

$\mathrm{Au}$ début du roman donc, il y avait le verbe. Le manifeste sorélien est celui du tout dire. L'Advertissement de 1623, s'appuie sur la franchise, mais également, en termes littéraires du temps, sur la naifveté d'écriture. L'argument sorélien est le suivant : si une chose existe dans le monde, nous avons légitimement le droit de la nommer et donc d'utiliser un processus sémiologique qui la désignera. Aussi le langage doit-il suivre le réel dans toutes ses vicissitudes, quand bien même un interdit social s'y opposerait. Sorel se demande avec une fausse ingénuité : " pourquoy ne sera t'il pas permis de dire des choses que l'on ose bien faire? J'ay trop de franchise pour celer la vérité $\gg^{14}$. Il y avait bien là un projet romanesque d'ordre mimétique que Sorel se devait, après déclaration officielle, de respecter. Il le fait le plus souvent possible, notamment face au problème de la retranscription de l'oralité, car au delà de l'observateur littéraire, Sorel est un observateur du fonctionnement de la parole orale. Il est à coup sûr ennemi de ce livre, "mon livre », confie Francion, " qui ne me disoit pas un mot plus haut que l'autre ${ }^{15} »$, et il laisse aux fluctuations de l'oralité une large place dans son roman, poussant cette fidélité jusqu'à la transcription précise du bégaiement : "Monstrez moy, enfin ce dis-je, monstrez moy un des beaux diamants que vous ayez ${ }^{16} \gg$. On repère dans le premier roman comique de Sorel des hésitations, des balbutiements, des pauses réfrénant la cadence des paroles ; ce discours romanesque « seems moreover to be punctuating by breath pauses $[\ldots]$ following not only syntax and sense but the patterns of oral speech ${ }^{17} \gg$. Le souci de transcription des moindres détails vocaux est même scrupuleusement respecté dans l'accent

\footnotetext{
${ }^{9}$ Ibid., tome II, p.186.

${ }^{10}$ Ibid., tome II, p. 216.

${ }^{11}$ Ibid., tome II, p. 130.

12 Ibid., tome I, p. 135.

13 Ibid., tome I, p. 132.

14 Ibid., tome I, p.IX.

15 Ibid., tome I, p. 172.

16 Ibid., tome I, p. 46.

17 Robin Howells, Carnival to classicism, the Comic Novels of Charles Sorel, Biblio 17, Papers on French Seventeenth Century Literature, Paris-Seattle-Tübingen, 1989, p. 34.
} 
dialectal des personnages, et l'on nous apprend par exemple que Francion ne se débarrasse de sa prononciation bretonne qu'à l'époque du collège ${ }^{18}$.

Une telle rigueur de reproduction du langage oral amène bien sûr l'auteur à se faire le témoin des différences sociolinguistiques - sur le plan des idiolectes comme de la phonétique. Le langage des conditions, comme l'a dénommé Hervé Béchade, se devait d'être lui aussi respecté - et il l'est, des cajoleries gauches des paysans aux pointes ridicules des poètes parisiens. C'est sur ce point précis, d'ailleurs, que Sorel prend parti dans une polémique littéraire dont il est, en ce début de XVIIème siècle, plus ou moins l'instigateur, à savoir le respect des réalités sociolinguistiques dans l'univers fictif du roman. Joconde, personnageprétexte, servant ici de porte-voix à Sorel, s'offusque au sujet des romans pastoraux : «les Bergers sont icy dedans Philosophes, et font l'amour de la mesme sorte que le plus galand homme du monde [...] Je suis d'advis pour moy que l'on compose un livre des amours de Chevaliers à qui l'on fasse parler le patois des paysans ${ }^{19} "$. Le langage n'apparaît pas comme un élément niveleur, mais bien au contraire comme le moyen le plus sûr de se marquer socialement et de se distinguer. Sur ce point, Francion est au-dessus de tout soupçon de vulgarité ${ }^{20}$ - vulgarité s'entendant dans sa double acception de grossier et de populaire.

Le débat qui a lieu entre le héros et Raymond, en pleine orgie libertine, reprend cette idée et l'approfondit sur le plan de la sémiologie. Si l'acte sexuel est différent chez les paysans et chez les vrais nobles, car « ils n'apportent pas neantmoins les mesmes mignardises et les mesmes transports d'esprits » affirme Francion, les mots doivent tenir compte de cette différence, et l'on sent la nécessité de mettre en place un double vocabulaire. A signifiés différents, signifiants différents. Les manifestations du corps n'ont donc pas, malgré les apparences, un effet anarchisant stricto sensu sur les hommes. « voicy ce que (Francion dit) là dessus » :

puisque les mesmes parties de nostre corps que celles du leur se joignent ensemble, nous devons remuer la langue, ouvrir la bouche et desserrer les dents comme eux quand nous en voudrons discourir [...] ainsi en discourant de ce jeu là, bien que nostre corps face la mesme action qu'eux pour en parler, nostre esprit doit faire paroistre sa gentillesse, et nous faut avoir des termes autres que les leurs. ${ }^{2}$

Il est à noter que cette réflexion constitue une évolution dans la pensée du héros, qu'elle se situe après l'expérience de son dépucelage frauduleux, et après la discussion dans la chambre de Raymond lors de laquelle l'ami de Francion avait conclu à une égalité de toutes les femmes en situation d'amour charnel. A l'acmé de la gaillardise du livre, Francion cimente ici son point d'arrivée dans la réflexion sur la sexualité et les signes. Comme tendra à le confirmer la fin de l'ouvrage, Raymond représente une dépouille, une chrysalide symbolique, des anciennes - et caduques - pensées du héros principal ${ }^{22}$.

Les nouvelles se concentrent sur une liaison, la plus intime possible, des mots et des choses. Dans cette optique entrent les quelques tentatives d'explication - souvent anecdotiques - des signes. Les poètes rebaptisent les parties de leur vêtement du nom des

\footnotetext{
18 « Je ne tenois plus rien du tout de nostre pays, non pas mesmes les accents » avoue Francion. Francion, tome I, p.181.

19 Ibid., tome III, p.127-128.

${ }^{20}$ Francion parle « avec une grace qui n'est point dans le vulgaire ». Ibid., tome II, p.65.

21 Ibid., tome III, p. 30.

22 Voir sur ce point notre article, « Les avatars de l'Histoire comique de Francion », Studi franncesi, anno XL fasc.III, settembre-dicembre 1996, p.511.
} 
livres qui leur a permis de se les procurer $^{23}$, Paris s'est appelé Lutèce à cause de sa boue ${ }^{24}$, Sorel remonte dans l'histoire des mots pour juger de leur bien-fondé. Rêver d'un idiome dans lequel les signes seraient fortement motivés - langage qu'appelaient de leurs vœux Cratyle et Socrate - est pourtant difficilement envisageable quand on emprunte un matériau collectif et ancestral, tel que le langage. Toutefois, il existe des possibilités pour motiver des signifiants. C'est le cas lorsque l'on crée ces signifiants ; et l'on crée des signifiants en créant des romans, des noms de personnages ou de lieux : le nom propre est bien souvent terre de prédilection pour un auteur à la recherche d'une motivation sémiologique. Tel se présente le patronyme d'Hortensius. Le nom réel du personnage, Le Heurteur, est l'anagramme approximatif de Le Rhéteur (approximatif car un $u$ est de trop), et si l'anagramme dit vrai, la faculté de rhéteur, de détenteur de la rhétorique, est intrinsèque à Hortensius. Voilà une idée qui s'accorde mal avec celle que Sorel se fait des pédants, qui sont des gens qui viennent " de la charruë à la chaire $^{25} »$, qui ne doivent leur privilège d'enseigner qu'à « la corruption de ce siecle ${ }^{26}$ » et non à une valeur personnelle. Il faut reconnaître, de plus, qu'Hortensius porte bien son nom de heurteur, car il heurte. Il violente ses élèves, mais il heurte également par sa sottise, son avarice, son pédantisme, les beaux esprits ainsi que ceux des collégiens. Retenons encore le sens de l'adjectif heurté pour qualifier un style langagier, "style dont les pensées ne se suivent pas ou se lient difficilement ${ }^{27} \gg$ - ce qui désigne parfaitement le discours que le pédant tiendra à Fremonde. L'anagramme est-il alors vraiment indispensable ? Concédons que toutes les hypothèses peuvent être concomitantes, qu'Hortensius peut se révéler un rhéteur heurteur, et que dans les deux cas le signe a été motivé en fonction de traits caractéristiques du personnage. Le nom usuel d'Hortensius est lui-même riche de sens, et fait référence à Q. Hortensius Hortalus, de Rome, rival de Cicéron pour l'éloquence. Ce dernier fait de lui un portrait globalement élogieux, mais quelques remarques laissent à penser que le personnage sorélien emprunte ses traits à l'orateur latin. Cicéron précise qu' «il reproduisait dans les mêmes termes exactement ce qu'il avait préparé en sa tête " (ce que le pédant fera à Rome, avec ses compliments préalablement écrits et cachés dans son chapeau), et que « ses attitudes et ses gestes trahissait plus d'artifice qu'il neût convenu à un orateur ${ }^{28} \gg$ (attitude outrée que l'on retrouve également dans les exagérations de l'ancien maître de Francion).

Mais cette adéquation du signifiant à son signifié, créée de toutes pièces par Sorel, est bien le négatif d'une réalité sémiologique observée par l'auteur. Les mots sont au-dessous des choses, doit-il admettre; du coup, son projet mimétique est mis à mal. L'expérience du portrait de Naïs est caractéristique sur ce point. La représentation picturale de la belle est imparfaite. Francion le remarque ${ }^{29}$ et Raymond le confirme ${ }^{30}$. L'épisode ne vaut pas que pour l'art pictural, mais plus généralement pour l'éloignement de la réalité et des moyens employés pour la représenter. D'ailleurs, c'est sur le constat d'une différence considérable entre le monde des mots et le monde du réel qu'Agathe justifie les libertés de son récit, au livre II du Francion. Elle se permet de raconter son existence au héros et à Raymond parce qu'euxmêmes ne sont pas « de ces delicats à qui un récit est d'aussi mauvaise odeur que la chose

\footnotetext{
${ }^{23}$ Un poète explique à Francion : « voila pourquoy pour recognoistre nos habillemens, nous les appelons du nom des Livres que nous avons faicts, et de l'argent desquels nous les avons eus ». Ibid., tome II p.91.

24 «il rien de plus commode pour espargner les bas de soye a qui les crottes font une guerre continuelle, principalement dedans Paris, qui a cause de sa bouë fut appellée Lutece ». Ibid., tome III, p.177.

${ }^{25} \mathrm{Ibid}$., tome II, p.4.

${ }^{26} \mathrm{Ibid}$., tome I, p.IV.

${ }^{27}$ Littré, entrée heurté.

${ }^{28}$ Cicéron, Brutus, 301-303, cité par Jean Bayet, Littérature latine, Armand Colin, Paris, 1965, p.119-120.

29 « Il luy estoit advis mesme que le peintre avoit oublié beaucoup d'attraits ». Francion, tome III, p.87.

30 «Lui qui ne l'avoit point encore veuë l'admira et la trouva plus belle qu'elle n'estoit en son portrait que l'on lui avoit monstré ». Ibid., tome III, p.166
} 
mesme $^{31} \gg$. Le personnage de Carmelin répétera avec plus d'instance encore, dans le Berger extravagant, la conviction sorélienne d'une barrière infranchissable entre le monde des signes et le monde réel : « de mesme que pour entendre parler d'huile \& de graisse, nostre robe n'en est point tachée, je croy aussi que les paroles ne puent point ${ }^{32} \gg$.

Conséquemment, l'auteur recherche des manifestations plus outrées d'une inadéquation des mots aux choses. Dans la panoplie des mensonges, il va jusqu'à débusquer des antiphrases lexicalisées, particulièrement dans le vocabulaire des magistrats ${ }^{33}$, et en termine sur le psittacisme. Les extravagances verbales d'Hortensius ou, plus encore, les élucubrations magiques de Valentin en sont des exemples patents. "Son seul secours fut de dire ces paroles ridicules qu'il avoit apprises pour sa defense : O qui que tu sois, grand mastin, qui accours à moy tout esbouluy, la queuë levée, pensant avoir treuvé la curée qu'il te faut, retourne t'en au lieu dont tu viens, et te contente de manger les savates de ta grand'mère . Il se figuroit qu'il avoit là dessous quelque sens magnifique de caché ${ }^{34} »$. Des mots vides de sens, voilà peut-être à quoi tend cette démarche de quête des aberrations sémiologiques. Le constat qui en résulte est que le réel s'appréhende directement et la fiction ne doit être envisagée qu'après un apprentissage direct. Le reproche que fait Francion à Hortensius est clair et sert de règle à suivre dans cette problématique humaine et didactique : "Le plus beau livre que vous puissiez voir, repliqua Francion, c'est l'experience du monde ${ }^{35} \gg$. Lysis sera un frère jumeau d'Hortensius sur ce plan-là. Si le jeune homme est devenu un berger extravagant, c'est qu'il n'a fait que lire depuis son enfance. Quand le langage n'existe que par rapport au langage et qu'il a perdu sa fonction mimétique, sa fonction de représentation d'une réalité déjà connue dans un rapport concret, Sorel réagit vivement et s'écrie : " de tels livres ne contiennent que langage ${ }^{36} \gg$. C'est une idée du temps, tout autant cartésienne que sorélienne. André Robinet nous rappelle que « Descartes s'inquiète plus loin du rapport fallacieux du mot à la chose, dès que l'entendement ne fait plus nettement attention à ce qui est désigné par le $\operatorname{mot}^{37}{ }^{3}$. C'est ainsi que l'on comprend la réflexion de Naïs sur l'amour qu'elle a porté au portrait de Floriandre. La représentation ne saurait être aimée en elle-même et Naïs affirme : « J'ay esté bien sotte jusques icy d'aymer une peinture, disoit elle en elle mesme ${ }^{38} »$. Pour Sorel, il ne saurait être question de vivre, comme Lysis, dans un monde bâti de mots seulement. Très vite, alors, l'entrée dans l'univers des récits est perçue comme un piètre succédané de l'expérience avec le concret. On se résout aux mots seulement à défaut du monde. Le récit du rêve de Francion n'est dû qu'au voyage en carrosse et à l'ennui que l'on peut éprouver en n'y parlant pas $^{39}$; l'explication de ce songe doit son existence à l'ennui que les héros ressentent lors du même voyage ${ }^{40}$; Agathe, enfin, n'entreprend la révélation de son existence que parce qu' « il

\footnotetext{
${ }^{31}$ Ibid., tome I, p.100.

32 Charles Sorel, Le Berger extravagant, éd. Hervé D. Béchade, Slatkine reprints, Genève, 1972, p.328-329 (266-267). (Le premier chiffre indique la page de l'édition Slatkine, le second, entre parenthèses, indique, à l'intérieur de cette première page, le numéro de l'une des quatre pages de l'édition originale du XVIIème siècle).

${ }^{33}$ Le bailly porte mal son nom puisqu'il « prend bien et ne baille guiere ». Francion, tome I, p.151. De même pour le Procureur qui procure «plus tost pour luy mesme que pour autruy ». Ibid., tome I, p. 155. On parle de chiquanerie pratique «sans dire de quoy elle est pratique ». Ibid., tome I, p. 158. Et l'on se demande enfin : «Qu'est-ce qu'un Greffier, [...] un homme qui jouë de la griffe ». Ibid., tome II, p.58.

34 Ibid., tome I, p.5.

35 Ibid., tome IV, p.61.

${ }^{36}$ Le Berger extravagant, op. cit., p.552 (10).

${ }^{37}$ André Robinet, Le langage à l'Age Classique, Klincksieck, coll. "Horizons du langage", Paris, 1978, p.81.

38 Francion, tome III, p.91.

${ }^{39}$ Donc, « tandis que le carosse rouloit à travers les champs, Francion commença ainsi à parler ». Ibid., tome I, p.122.

40 « Toutefois si par maniere de passetemps vous trouvez bon que je philosophe sur ce songe », propose Raymond. Ibid., tome I, p.146.
} 
fait si mauvais temps que ne pouvant encor sortir d'icy il nous faut quelque entretien ${ }^{41}$ ", indique-t-elle.

\section{II. « Je veux faire ce qui n'est jamais entré en la pensée d'un mortel » (Francion, 1626 : livre XI ${ }^{42}$}

C'est dans un climat d'insatisfaction littéraire que Sorel a écrit une décennie durant, de la première mouture du Francion, en 1623, à la dernière du Berger extravagant, de 1633-34, dont le nouveau titre sera précisément l'Anti-roman. L'unité des deux textes comiques sur le plan de la critique métalittéraire ne fait pas de doute ${ }^{43}$. On y remarque la volonté d'en finir avec une poétique, tout en la conspuant. Lysis et Hortensius, principalement, sont les agents et les acteurs de cette avanie. Le pédant du Francion prend sur lui les habitudes et les erreurs littéraires des écrivains maudits par Sorel. Acrostiches, Anagrammes ${ }^{44}$, discours inutilement érudits, tout ce que produit Hortensius sert, par effet intertextuel, à railler les écrivains. Le phénomène est encore plus symptomatique en ce qui concerne son langage tropologique. Francion critique Hortensius parce que ses «comparaisons sont trop esloignées ${ }^{45}$ ». Le narrateur du roman comique poursuit quatre pages plus loin : "Là dessus il usa de tant de termes extraordinaires, que Francion ne les pût d'avantage souffrir sans lui demander s'il faloit parler comme il faisoit, veu qu'il n'avoit rien en son stile que des hyperboles estranges, et des comparaisons tirées de si loing que cela ressembloit aux resveries d'un homme qui a la fievre chaude ou au langage de l'Empereur des petites maisons ${ }^{46} \gg$. Le stile d'Hortensius est bien la voie poétique sur laquelle s'engagent de plus en plus d'auteurs du temps ${ }^{47}$; que ce soit pour leurs poèmes obscurs, galimatias et autres coq-à-l'âne, ou pour leur prose, comme celle de Balzac que le pédant reprendra presque au mot près à Rome.

Ce n'est pourtant pas seulement à cette fonction de représentativité du contexte littéraire que l'on doit rattacher le personnage de Collinet. Des galimatias, certes le fou de Clérante en propose, mais il faut y regarder de plus près. Plus spécifiquement ses tirades sont des collages de certaines images rencontrées dans les recueils de vers satiriques, en particulier

\footnotetext{
41 Ibid., tome I, p.62.

42 Ibid., tome IV, p.8.

43 Cette idée d'unité, Sorel la confirme à l'intérieur même de sa production artistique. Dès le Francion de 1626, il annonce son Berger extravagant dont il attribue la paternité à son héros éponyme, et met ainsi en place une structure dialogique qui unifie l'ensemble des récits soréliens que l'échelonnement de publications distinctes sépare. C'est certainement moins Francion parlant à ses amis immédiats que Sorel à son public, que nous observons dans ces quelques lignes de l'Histoire comique : «Or pour vous parler de ce dernier livre que je n'ay pas escrit, mais que j'ay seulement en l'imagination pour ce que je portois la houlette lors que j'y ay songé, son titre sera Le Berger extravagant. Je descry un homme qui est fou pour avoir leu des Romans et des Poesies, et qui, croyant qu'il faut vivre comme les Heros dont il est parlé dans les livres, fait des choses si ridicules qu'il n'y aura plus personne qui ne se moque des Romanistes et des Poetes si je monstre cette Histoire ». Ibid., tome IV p. 29-33. Sorel tient à cette invention et insiste plus loin pour lier structurellement les deux ouvrages dans la même entreprise. Pour ce faire, il altère la fiction d'un Francion rédacteur du Berger, et se propose directement comme l'ordinateur des compositions de son héros. Tel un pont entre les livres, il place cette promesse de travail unificateur en clausule du Francion de 1626 : «En attendant je travailleray a mettre par ordre les avantures du Berger extravagant que Francion a composées, et les donneray au public comme une seconde partie de ceste Histoire Comique ». Ibid., tome IV, p. 72 .

44 Dès le début du livre XI, l'ancien professeur du héros « fit des Acrostiches, et des Anagrammes comme estant chose fort propre a son Genie pedantesque ». Ibid., tome IV, p.24.

45 Ibid., tome IV, p.3.

${ }^{46}$ Ibid., tome IV, p.7.

47 Hortensius ressemble à un poète lorsqu'il cherche trop loin les phores de ses analogies - «pour faire des pointes à la façon de tous les escrivains, qui raportent souvent pour comparaison des choses qui ne furent jamais », comme on devra le lire dans le Berger extravagant, op. cit., p.633 (333).
} 
ceux de Sigogne et de d'Esternod ${ }^{48}$. Mais au delà, son discours est une violente attaque hétérodoxe pour qui sait décoder les circonlocutions, ainsi que Pierre Ronzeaud l'a montré ${ }^{49}$. Et au delà encore, le langage de ce fou-morosophe est une rupture savante avec une certaine convention tropologique, lors de l'évocation de Luce en particulier. La métaphore des cheveux d'or des jeunes filles qui hante la littérature depuis l'Antiquité, en passant par Iseut ou l'Enide de Chrétien de Troyes, est mise à mal par le fou de Clérante, et Collinet brise une tradition tout autant qu'une technique de métaphore ou de comparaison : " vos cheveux sont jaunes comme la merde d'un petit enfant» dit-il à Luce ${ }^{50}$. Il en va de même pour les descriptions de la joie, car pour le personnage on peut être " ravy comme un pourceau qui pisse dans du son ${ }^{51} "$, Collinet prouvant par là sa liberté à opérer des rapprochements, à chercher un effet jusqu'alors insoupçonné par les conventions langagières. En tout état de cause - et c'est ce que Sorel veut prouver - les images de Collinet, si surprenantes soientelles, demeurent plus acceptables à un esprit rationnel que celles des poètes. Une fois de plus le mensonge, mais ici via les tropes, est montré du doigt.

Toute la surenchère verbale, tendant à faire de la belle aimée une autre déesse, est moquée intrafictionnellement. Dans le Berger, la vue d'une femme sous les draps prouve que l'être féminin n'est pas divin ${ }^{52}$, épisode qui fait écho aux sarcasmes de la servante d'Agathe, qui rétorque au transi Valdéran que ses chansons qui peignent sa maîtresse telle une déesse n'ont pas vu les détails corporels indissociables de la belle : "Vous nous la baillez belle, dit ma servante », raconte Agathe, " vous prenez donc Laurette pour une Déité ? Voulez vous voir ce qui est dans sa chaire percée, et si vous aurez bien le courage d'en manger : Ce n'est point du Nectar ni du Maistre Ambroise ${ }^{53}$ \». Trois ans plus tard, en 1626, Sorel poursuit son invective et établit de la même manière un parallèle entre les tropes alchimistes des poètes et leur référent sordide du réel ${ }^{54}$.

Que l'on rétorque à Sorel qu'il ne s'agit là que d'une allégorie et l'argument sera de peu de valeur à ses yeux. Rien n'est plus porteur de mensonges, en effet, que l'allégorie. Loin d'être le véhicule d'une vérité unique et indéniable, la figure de style, en pouvant tout dire, finalement ne dit plus rien de fiable. Quand Lysis voudra trouver dans le décor et la vie qui l'entourent des signes allégoriques de ses amours, le narrateur précise :

il voulut esprouver quelque presage afin de sçavoir la fin de ses amours. Il s'en alla en un lieu où il trouva une servante qui chauffoit le four, \& l'estant venu dire à son maistre, il luy aprit que cette fille representoit Lisette qui avoit mis le feu en sa poictrine, \& que pour le pain qu'elle y mettoit cuire, cela vouloit monstrer, que cette belle participeroit à ses ardeurs, \& luy doneroit son cœur à eschauffer. ${ }^{55}$

\footnotetext{
48 Voir notre étude, Romans comiques et romans satiriques sous Louis XIII : une question de langage, Thèse de Doctorat, Université de Provence, 1997, p.204.

49 Voir Pierre Ronzeaud, «Parole libertine et discours de fou : le rôle de Collinet dans le Francion de Charles Sorel (1623) », L'Intelligence du passé, les faits, l'écriture et le sens, Mélanges offerts à Jean Lafond par ses amis, Publications de l'Université de Tours, Tours, 1988, p.257-265.

${ }^{50}$ Francion, tome II, p.152.

51 Ibid., tome II, p.153.

52 «En fin celluy-cy conclud qu'il ne sçauroit dire tant de fables sans mourir de rire, \& qu'il dira bien à n'en point mentir, que cette belle Dame est en bon poinct dessous les draps, mais que ce seroit une resuerie de dire qu'elle est divine ». Le Berger extravagant, op. cit., p.571 (88).

53 Francion, tome I, p.111-112.

54 « Ma foy, ce dit alors Francion, il me semble que j'entend encore les fables de ces fees dont les servantes entretiennent les enfans. L'on dit que si elles alloient a la selle, elles n'y faisoient que du musc; si elles pissoient c'estoit eau d'ange ; si elles crachoient ou si elles se mouchoient, il sortoit de leur nez et de leur bouche des esmeraudes et des perles : si elles lavoient leurs mains, au lieu de crasse, il en tomboit aussi des pierres precieuses ». Ibid., tome IV p.14.

55 Le Berger extravagant, op. cit., p.459 (790).
} 
Le ridicule de la recherche et surtout de la découverte de symboles dans les scènes ou les objets les plus prosaïques vaut pour désacralisation du procédé allégorique. Bref, Sorel est en train de prouver, par l'expérience de son héros fou, que l'on peut trouver du sens à n'importe quoi, et - plus grave encore - n'importe quel sens à n'importe quoi ${ }^{6 \sigma}$. Que ce soit un insensé qui découvre cela n'est pas sans intention profonde de la part de l'auteur. Aussi, la critique sorélienne tombera comme une sentence de mort $^{57}$ sur l'allégorie dans le Berger extravagant: « on treuve tout ce que l'on veut par Allegorie dans quelque narration que ce soit, \& [...] à un mesme suject un esprit inventif peut donner dix mille explications $\|^{58}$. De là la raillerie, d'Hortensius cette fois, d'une allégorie se réduisant finalement pour les personnages du Francion à de simples jeux de mots :

il se mit sur les loüanges de la Musique et asseura que les passions et les affections humaines en representoient les parties. L'humilité chante la basse, disoit-il, et l'ambition chante le dessus, la colere fait la taille, et la vengeance la contre-taille, la modestie tient le tacet : la Prudence bat la mesure, et conduit le concert : la Nature va le plein chant : l'artifice fredonne : la douleur faict les soupirs, et la dissimulation les feintes et les dieses. Et pour les instruments de Musique, l'avarice joüe de la harpe : la prodigalité joüe du cornet a jetter les dez : l'Amour joüe de la viole, pource qu'il fait violer les filles : la trahison joüe de la trompe, car elle trompe tout le monde : et la justice joüe du hautbois, pour ce qu'elle fait elever des potences pour y attacher les coulpables..$^{59}$

Au delà des jeux de mots, c'est toute la technique des réseaux allégoriques qui est raillée et contestée de l'intérieur. Par ces figures de style Sorel ne trouvera ainsi pas le vrai - ou le neuf.

Confiant dans la démystification que le corps est toujours prêt d'apporter à la fiction des poètes ou de tout travestissement ${ }^{60}$, la matière fécale en particulier (la diarrhée de Carmelin interrompra une pièce de théâtre dans le Berger), l'écrivain parisien cherche du même côté des visions déformées de la réalité mais non moins indéniables. Le problème devient donc plus complexe, dans la mesure où il propose une réflexion sur la conception initiale qu'un individu se fait du monde, avant même de penser à une altération postérieure par un verbe modificateur. On se souvient, par exemple, de la remarque de Francion à propos des excréments trouvés dans le banc de Diane, à Saint Séverin : "la veuë de ceste infection me plût davantage que n'a jamais fait celle des plus belles fleurs ${ }^{61} »$. Le personnage éprouve une joie excessive à la vue des matières fécales parce que celles-ci prouvent que Diane était partie de l'église, non pour fuir la présence de Francion, mais pour l'odeur insupportable de l'ordure. Ainsi, à une vision habituelle et juste d'un élément du monde (et ici c'est l'excrément, qui avait pourtant rappelé à Valdéran la véritable nature humaine de Laurette), le héros lui-même substitue une autre appréciation en vertu d'un autre critère (l'amour de Diane, qui n'est donc pas remis en question). C'est tout le problème de l'interprétation exacte et objective

\footnotetext{
56 L'idée se retrouve dans le théâtre de Corneille. Serge Gaubert note : «Les mots et les choses jouent leur propre jeu, dans leur propre domaine. Tout peut, à la limite, servir à qualifier n'importe quoi ». « De la comédie des signes aux signes de la comédie », Europe, n ${ }^{\circ} 40-541$, avril-mai 1974, p.79.

${ }^{57}$ Nous reprenons cette image à Dalia Judovitz et au thème central de son article « Mort de l'allégorie dans Le Berger extravagant $»$, Lendemains, $\mathrm{n}^{\circ} 13$, Etudes comparées sur la France, Berlin, 1988, p.46-55.

${ }^{58}$ Le Berger extravagant, $\mathrm{p} .500$ (53-54)

${ }^{59}$ Francion, tome IV, p.69.

${ }^{60}$ La fiction que le voleur-Catherine essaie d'instaurer sur la qualité de son sexe ne résiste pas à l'appel de la chair ; le brigand travesti « voyant le beau sein de Laurette tout descouvert, fut chatouillé [...] de desirs un peu plus ardents que ceux qui pourroient esmouvoir une personne de sa robbe ». Ibid., tome I, p. 18.

${ }^{61}$ Ibid., tome II, p.111.
} 
qu'élaborent les hommes sur le monde. En ce sens, le héros sorélien propose une vision particulière du monde en vertu d'une synesthésie. Cette synesthésie, dans la mesure où elle associe un sentiment particulier - ici le plaisir - à un élément du monde qui lui est diamétralement et habituellement opposé, remet en cause les visions traditionnelles, du moins dans leur fonctionnement.

A cette lumière, on comprend l'intérêt de Sorel pour les visions particulières qu'un esprit altéré (par l'alcool ou la folie) engendre. Hortensius redouble de stupidités verbales quand on le fait boire ; Collinet « par le moyen de deux ou trois verres d'un vin de singe ${ }^{62}$ » est prêt à débiter son galimatias ; Clérante invitait des villageois « et se plaisoit à les voir boire d'autant afin qu'ils contassent après merveilles ${ }^{63} »$; les exemples sont nombreux : Francion qui compose durant ses débauches ${ }^{64}$, ou les extravagances langagières du suisse ivre. C'est d'ailleurs dans ses moments de folie qu'Hortensius se voit réhabilité par la compagnie. Le personnage est alors double. D'un côté il est un plagiaire, comme les autres membres du milieu universitaire, de l'autre il fait montre d'un vrai talent d'invention. D'un côté et de l'autre... ou plutôt d'une édition (1623) à l'autre (1626). Dans le premier état du texte, les pédants et autres férus d'érudition latine sont victimes d'une véritable sclérose créatrice, car « n'ayant rien à donner d'eux-mêmes, ils en sont continuellement réduits à emprunter aux autres $^{65} \gg$. C'est encore au siècle précédent qu'il faut recourir pour retrouver les racines de cette critique, dans un XVIe siècle où déjà « Louis Le Roy ${ }^{66}$ rejetait l'imitation comme étant le fait des "paresseux et lasches de cœur" "ㄱ ». Dans le Francion, ces «lâches de cœur » sont le Régent du Collège, qui « tranchoit grandement du Poëte ${ }^{68} 》$, mais qui doit emprunter, pour ses forfaits littéraires, aux « Comédies imprimées, dans la farce de Pathelin et dans le Romant de la $\operatorname{Rose}^{69}$ " ; Hortensius qui se sert du De Amore, des passages sélectionnés de celui qu'il nomme le «Ciceron françois », pour séduire sa bien-aimée ; le Comte de Bajamond qui, " quand il alloit en compagnie il apprenoit par cœur quelque discours, qu'il tiroit de quelque livre, et le recitoit, encore que l'on ne tombast aucunement sur le subjet ${ }^{70}{ }^{\prime}$; ; les poètes de la librairie Saint-Jacques, qui ont bien vite lassé Francion, dont le regard a été assez juste pour s'apercevoir que « toutes leurs inventions estoient imitées ${ }^{11} »$. Sorel ne méprise pas l'imitation, mais seulement ceux qui en font une fin et non un moyen ; ne nous avoue-t-il pas que son jeune héros s'y adonne afin de parfaire sa plume ? ${ }^{72}$ Mais cette étape d'apprentissage a vocation à être dépassée. Le dépassement aura lieu pour Hortensius au livre XI et trois ans plus tard. Le pédant propose une multitude d'histoires qui, si extravagantes soient-elle, demeurent susceptibles d'être rédigées. Un roman dont l'action se déroule dans l'espace, un

\footnotetext{
62 Ibid., tome II, p. 150.

63 Ibid., tome II, p. 175.

${ }^{64}$ Francion parle de ses chansons : « les plus folastres [...] que j'avois composées le plus souvent le verre à la main, pendant mes desbauches ». Ibid., tome II, p. 186.

65 Frank-Edmund Sutcliffe, Le Réalisme de Charles Sorel, Problèmes humains du dix-septième siècle, Nizet, Paris, 1965, p. 173.

66 C'est-à-dire Loys Leroy, auteur du traité De la vicissitude ou variété des choses en l'univers, Paris, 1583. Voir bibliographie in Ibid., p.215.

67 Ibid., p. 183.

68 Francion, tome II, p.4.

69 Ibid., tome II, p.6.

${ }^{70}$ Ibid., tome II, p. 214.

${ }^{71}$ Ibid., tome II, p.80.

72 Lors des répétitions théâtrales, au collège, Francion confie : « lors que j'en joüay mon personnage, il n'y avoit rien qui ne me semblait extremement bien faict, et je taschois d'en imiter les vers, lorsque $\mathrm{j}$ 'en voulois composer d'autres ». Ibid., tome II, p.5-6. Plus tard, une fois les portes du collège passées, le héros s'intéresse à la poésie : « je vous conte que l'on me mit en main quelques ouvrages assez polis, sur lesquels je façonnay ceux que je fis par après ». Ibid., tome II, p.75.
} 
autre dans l'infiniment petit, un autre sous les eaux, un autre mettant en jeu les différentes parties du corps humain... De cette façon, par l'intermédiaire de projets fantasques ou sérieux, Sorel s'attache à prouver qu'il est aisé d'inventer, à condition de vouloir s'exempter de cette pratique d'imitation qui, selon l'auteur parisien, inhibe la création littéraire, rejoignant par là la vision de Théophile de Viau.

Mais les mortels comprendront-ils ? Sorel, quoi qu'il en dise, demeure soucieux de la réception de son discours. Avertissement en 1623 d'un décodage à effectuer pour certains passages du livre, impossible compréhension des plaintes amoureuses pour qui n'a jamais connu l'amour ${ }^{73}$, ou tout simplement bêtise «des ignorans qui ne pourront pas penetrer jusques au fond ${ }^{74} »$, autant d'obstacles que redoute l'auteur, et qui aboutissent à l'établissement de certains happy few auxquels seraient véritablement destinés les propos tenus ou le texte lui-même. On note cette idée chez Sorel ${ }^{15}$, dont se réclament, dans le Francion, le héros éponyme ainsi qu'Agathe. Dès le livre I, Francion dit à Raymond : «Encore qu'il semble que l'on devroit celer tout cela, librement je vous le descouvriray, d'autant qu'il m'est aisé à voir, que je ne puis confier mon secret plus asseurement ${ }^{76} »$. Si Francion a reconnu la valeur de son destinataire langagier au seul aspect physique, force nous est de constater qu'ici choix d'un interlocuteur privilégié et physiognomonie s'entrecoupent. Agathe, elle, narre ses aventures à Francion et Raymond car elle sait que leurs « esprits ne sont pas faicts de cruche, comme ceux des autres ${ }^{77} \gg$. On comprend ainsi mieux la présence presque systématique de narrataires intrafictionnels dans l'Histoire comique de Francion (c'est avant tout le rôle de Raymond).

Ceux-ci considèrent d'ailleurs les récits de leurs interlocuteurs comme un paiement verbal. Francion s'en réjouit à propos de Raymond : «Ce m'est un grand bonheur d'avoir rencontré un homme qui ne veut que des paroles pour recompenses des plaisirs qu'il me depart $^{78} \gg$. Plus jeune, le héros gagnait sa pitance à l'aide d'épigrammes ${ }^{79}$. Hortensius exagérera le processus en imaginant une monnaie poétique. Pour le pédant, la littérature gagne ainsi une valeur marchande, un pouvoir sur le concret, qui révèle tout autant un danger de subversion du langage qu'un questionnement sur l'action réelle des mots sur le monde. Car à quoi pouvait même servir un roman comique?

\section{III. « N'est il pas vray que c'est une tres agreable et tres utile chose que le stile comique et satyrique »? (Francion, 1633 : livre X) $)^{80}$}

A condition qu'il fût satirique, comprenons accusateur, le texte permettait-il la profération d'une médisance compensatoire, légitime riposte aux injustices du temps. Cette fonction générale du langage se retrouve çà et là dans l'Histoire comique de Francion. Aux

\footnotetext{
73 Lorsqu'on surprend Francion en train de se plaindre de la douleur amoureuse, il signale: « je suis marry que vous ayez entendu ce que j'ay dit : car si vous n'avez point esprouvé la force de l'amour, vous prendrés tout pour des folies les plus insignes du monde ». Ibid., tome III, p.85.

74 Ibid., tome I, p.IV.

${ }^{75}$ C'est aussi ce que pense Yves Giraud : « Les vérités fines ainsi soustraites à la foule des médiocres, Sorel les réserve aux happy few dont l'esprit est éclairé et délié ». Francion, éd. Yves Giraud, Garnier-Flammarion, Paris, 1979 ,p. 17.

76 Francion, tome I, p. 45.

77 Ibid., tome I, p.62.

78 Ibid., tome I, p.148.

${ }^{79}$ Francion narre ses aventures de Collège : « Nous estions aux nopces lorsque le Principal qui estoit un assés brave homme, festoyoit quelques uns de ses amis : car nous allions sur le dessert presenter des Epigrammes aux conviés, qui pour récompense nous donnoient tant de fruicts, tant de gasteau, et de tarte, et quelquefois tant de viande [...] Les meilleurs repas que j'ay pris chez les plus grands Princes du monde, ne m'ont point esté si delicieux que ceux que je prenois après avoir fait ceste conqueste par ma Poësie ». Ibid, tome I, p. 177-178.

${ }^{80}$ Ibid., tome III, p. 125.
} 
affronts de leurs maîtresses, Agathe et quelques autres servantes répondent par la médisance ${ }^{81}$; à la malice du siècle et les tortures de l'amour pour Diane, Francion répond par des vers ${ }^{82}$; aux fats qui prennent « la noblesse à l'habit », le héros répond par l'avanie ${ }^{83}$. Mais s'agit-il là d'un fantasme sorélien ou d'une certitude quant à la praxis du langage ? Le doute plane. Les poètes ne sont pas des hommes d'action, dans le roman; ils sont plus fréquemment empêtrés dans des questions financières ou linguistiques que dans une réalité historique. Aussi, raille-t-on généralement les compétences linguistiques qui n'ont que peu d'effet réel au niveau politique et collectif. Par moquerie, Raymond précise les qualités qui ont poussé les pseudo-Polonais à choisir Hortensius pour leur roi : «je m'asseure qu'une des raisons pour laquelle ils vous ont fait leur Roy, est qu'ils ont sceu que vous estiez bon Grammairien Latin $^{84} \gg$. Inversement, Sorel note combien une action vertueuse vaut mieux qu'un langage poli et agréable. Dès la préface du Francion, il revendiquait cette suprématie des actions sur les paroles. Il affirme : « ayant tousjours fait plus d'estat des actions que des paroles, j'aimerois beaucoup mieux m'exercer a la vertu qu'a l'eloquence ${ }^{85} \gg$. Cette assertion est à replacer dans le cadre de la réflexion sémiologique de l'auteur. Quand l'éloquence n'est aimée que pour elle-même, quand l'écrivain s'inquiète avant tout de son style, quand l'écriture seule a regroupé vers elle les préoccupations des auteurs, Sorel ne voit plus l'intérêt d'écrire.

A contrario, le Francion est un texte engagé, dans une réalité concrète, et il trouve là sa raison d'être. Le phénomène est certainement plus visible dans le premier état du roman. En 1623, l'auteur parisien prend parti dans une polémique des mœurs. Evoquer les pratiques sodomites dans les collèges, ou la masturbation des jeunes filles, ne revient pas à les mentionner en toute neutralité. Sorel, par l'entremise de son héros, en tire des analyses bien partiales :

un tel martyre me cause de la pitié, et je maudis les loix du monde, qui gardent que les remedes n'y soient donnez, et que tant de filles qui d'ailleurs souspirent en cachettes apres les embrassemens, ne soient mises avec ceux qui sont ainsi travaillez, afin qu'ils esteignent ensemble leurs flames par une eau la plus douce de toutes, et que desormais ils s'abstiennent de pecher. ${ }^{86}$

L'artisan du péché est bien l'univers moral dans lequel évoluent les jeunes gens du début du XVIIème siècle. On est loin ici des préoccupations linguistiques et esthétiques d'Hortensius ou des poètes parisiens.

En adoptant ainsi ce stile comique et satyrique, Sorel s'engage et (re)définit dans le même temps le rôle de l'écrivain. Le genre est caractéristique des options choisies par l'auteur : la même année 1623, qui voit paraître deux histoires comiques, le Francion mais également la Première journée de Théophile de Viau, montre à quel point une idée commune de la fonction de l'écrivain est en train de se dégager. De façon théorique, Théophile et Sorel sont d'accord pour revendiquer une totale liberté par rapport au goût du public, qui ne doit pas induire ou influencer la composition. Sorel écrit en préface du Francion de 1626 : Si "vous ne trouviez rien dans mon livre qui vous plaise, qui que vous soyez, Lecteur, ne le lisez pas

\footnotetext{
81 «C'estoit alors que nous nous entredisions nos infortunes, et que nous sçavions bien dire autant de choses de ces Madames, qu'elles en sçavroient dire de nous ; c'est un souverain plaisir que de mesdire, lorsque l'on est offencé : aussi ne nous y espargnions nous pas », nous confie Agathe. Ibid., tome I, p.65.

${ }^{82}$ Francion avoue : « Mon entretien ordinaire fut de composer des vers, sur la haine que je portois à la malice du siecle, et sur l'amour que j'avois pour la gentille Diane ». Ibid., tome II, p. 70.

83 «Quant à moy, tournant sa fascherie en risée, je recommencay à le brocarder», narre Francion à propos du jeune homme bien vêtu, dans la taverne. Ibid., tome II, p.119.

${ }^{84}$ Ibid., tome IV, p.38.

${ }^{85} \mathrm{Ibid}$., tome I, p.XVIII.

${ }^{86}$ Ibid., tome II, p.48-49.
} 
deux fois, aussi bien n'est ce pas pour vous que je l'ay faict. Ne l'acheptez point, je ne m'en soucie pas, si ce n'est pour l'interest du Libraire ${ }^{87} \gg$; et Théophile avait fini le premier chapitre de sa Première journée par ces mots : "Mon livre ne pretend point d'obliger le lecteur, car son dessein n'est pas de le lire pour m'obliger, et, puis qu'il luy est permis de me blasmer, qu'il me soit permis de luy deplaire ${ }^{88} \gg$. Il ne s'agit donc plus de plaire, mais de formuler tout ce à quoi la franchise se doit. Le rapport de séduction de l'auteur à son public est rompu au profit de l'engagement des auteurs dans une polémique (d'ordre social le plus souvent). Il faut rendre justice, avant que de songer à transporter le lecteur dans l'univers du roman ou pastoral ou chevaleresque ou héroïque, contre lequel le texte comique prend parti.

$\mathrm{Du}$ coup, Sorel responsabilise très fortement la démarche de l'écrivain qui rend compte, par les mots, de l'état du monde. Narrer les vicissitudes des prostituées et des voleurs de Paris entre dans cette optique vériste. Mais l'auteur regarde vers le bas comme vers le haut du corps social - ce qui le différencie des écrivains picaresques. Même les gentilshommes passent au jugement sorélien, le souci demeurant de louer ce qui est bon et de honnir ce qui est vil. Aussi, le héros lui-même est puni pour un éloge immérité, comme il le reconnaît luimême au sujet de Praxitele : « si le Ciel ne me favorisa en ce que je pretendois, ce fut pour me punir d'avoir loüé une personne indigne de loüange ${ }^{89} \gg$.

C'était faire l'économie du conditionnement mondain et courtisan de la parole au début du XVIIème siècle. Le souhait de tout dire librement, d'ailleurs, aboutit vite à un constat d'échec. L'écrivain est assujetti, comme les autres, au contexte social ; il en fait l'aveu amer, en 1626, malgré sa très provocatrice (et fausse) dédicace Aux Grands :

il faloit tascher de ne desobliger personne, et se rendre d'une humeur douce et complaisante, principalement à la Cour où il y a des esprits mutins qui ne sçauroient souffrir que l'on leur dise leurs veritez. ${ }^{90}$

Tout ce que peut alors réclamer Sorel est de ne point participer aux mensonges mondains. «Laissons là les sottises du temps, et qu'elles soient loüées de qui elles pourront,cela n'importe, pourveu que l'on ne nous contraigne point de les loüer aussi ${ }^{91}>$, lit-on dans le Francion. Par ricochet extrafictionnel, Sorel prône un détachement par rapport aux pratiques encomiastiques, qu'il relègue aux mêmes hypocrisies voire aux mêmes assujettissements. Le héros éponyme sert une fois encore de double de l'auteur et Francion s'en veut d'avoir dédié « des Livres a des stupides » et « donne au Diable et le Livre et le Seigneur, et protest(e) de ne faire plus de telles sottises ${ }^{92} \gg$.

Une réflexion sur les possibilités d'existence de la liberté de parole est alors amorcée. L'occurrence la plus intéressante, sans nul doute, se trouve dans le songe du personnage principal ; l'image du vieillard à la bouche cadenassée dans le rêve de Francion est peut-être la désignation d'une certaine répression religieuse affectant la liberté de parole, comme l'a démontré Wim De Vos : «Nous croyons que le tyran pourrait représenter la tradition jésuite de l'éloquence. Dans l'Imago primi seculi (1640), une image plus ou moins analogue figurera l'obéissance du religieux à son supérieur : "Pour rappeler qu'un Jésuite doit n'avoir aucun secret pour son supérieur, un cadenas qui s'ouvre grâce à un mot que seul le supérieur

\footnotetext{
${ }^{87}$ Ibid., tome I, p.XXI.

88 Théophile de Viau, Première journée in Euvres complètes, éd. Guido Saba, ed. dell'Ateneo-Nizet, RomeParis, tome II, 1978, p.17.

${ }^{89}$ Francion, tome II, p.172.

90 Ibid., tome II, p.225.

91 Ibid., tome IV, p.22.

92 Ibid., tome II, p.100.
} 
possède" ${ }^{\prime 3}$ ». Par le cadenas du vieillard, Sorel veut peut-être aussi nous inviter à la recherche d'un anagramme, puisque l'agencement des lettres est mouvant ${ }^{94}$. De plus, trouver le code magique, c'est libérer la parole du vieillard et donc découvrir ce qu'il a de précieux à dire. On pourrait cependant s'en arrêter à la phrase : il est temps, riche de sens. Les censeurs supposés, qui ont placé ce cadenas sur la bouche du vieillard, ont pris soin de ne lui donner la parole que quand il est temps - la parole est tenue en bride et l'on ne doit point s'en servir en dehors du temps imparti. De plus, il n'est pas exclu que le vieillard soit un personnage parodique, singeant les traditionnels prophètes - bibliques ou non - annonçant, en une énigme peu déchiffrable, le devenir de l'humanité. Voltaire se plaira à ce genre d'effet parodique, mais ici cette annonce d'un Temps qui arrive est à mettre en relation avec celle d'un Temps qui finit, et avec l'épisode de l'homme détruisant le pommier (puisque le pommier est amené à disparaître). Si l'on part de l'image de la pomme, autrement dit le péché originel, c'est évidemment l'arrivée du temps de la rédemption, qui annule - comme l'homme malicieux casse les branches - le péché originel. La brimade langagière affecterait ainsi la parole religieuse même, en empêchant le vieillard d'annoncer le temps de la rédemption!

La censure, la réduction au silence, relèvent bien d'une phobie chez Sorel. De là peut se comprendre le réflexe inverse de prolixité de l'écrivain, tout au long de sa vie ; de là également la probable idée que le texte demeure le dernier rempart derrière lequel on peut encore s'adonner à la divulgation d'un langage libéré et agissant. Le roman fait souvent fonction de contrario de la réalité. Alors que Sorel constate de plus en plus le pouvoir et l'étendue des mensonges, on trouve dans le texte une défaite de l'illusion, du paraître, au profit de la vérité. Le topos baroque du theatrum mundi y est mis à mal. Retenons que l'épisode de Francion travesti en berger parmi les paysans est long, mais il prend fin par la lassitude que le héros ressent à jouer un personnage qui n'est pas le sien. Francion, en effet, désire un jour «se retirer du village où il commençoit de se deplaire parmy les esprits grossiers, et ne desiroit pourtant se tenir en cet estat que pour une passade ${ }^{95} \gg$. On peut déduire par là que le carnavalesque, la dissimulation de la vérité par un monde artificiel, n'a de pouvoir que temporaire. Francion ne fait pas durer longtemps, non plus, la supercherie de se faire passer pour Floriandre, l'amant pour lequel Naïs est venu en France. Elle ne tient même que deux pages, et le héros s'efforce de reporter ses intentions carnavalesques sur une incompréhension de la part de Naïs ${ }^{96}$. Les supercheries d'Hortensius sont dévoilées, les machinations de Bajamond déjouées et montrées du doigt, même la cachotterie de Francion son aventure amoureuse avec Emilie - l'amène symboliquement en prison comme le remarque Naïs.

Elle dit qu'elle en recevoit de la satisfaction, et que c'estoit une punition manifeste qu'il recevoit de la part du Ciel, parce que s'il n'avoit falsifié les monnoyes, au moins

\footnotetext{
${ }^{93}$ Wim De Vos, « Corps constitués et corps monstrueux : allégories de la rhétorique chez Charles Sorel », p.1112.

94 En étudiant $i l$ est temps comme un anagramme, on peut découvrir il tempeste (anagramme approximatif puisqu'il nécessite le rajout d'un $e$ et la suppression d'un $s$ ). La découverte serait alors prospective de la colère du géant qui, en effet, tempeste contre les langues et le rocher. Notons que Sorel connait et emploi ce verbe tempester , connoté, selon lui, d'une grande agitation : « L'un crie, l'autre tempeste, l'autre court » écrira-t-il au livre IV. Un autre anagramme - anagramme précis cette fois-ci est encore possible : tes mil pets. Celui-ci serait prospectif de l'odeur nauséabonde qui sortira des entrailles du géant. L'hypothèse est hasardeuse, d'autant que l'on ne saurait pourquoi le cadenas indiquerait un possessif à la deuxième personne. Restent encore deux possibilités d'anagramme : mes petits $l$ ou bien les petits $m$. Que seraient $l$ ou $m$, et pourquoi seraient-ils petits ? ${ }^{95}$ Francion, tome III, p.13.

96 «Francion voyant alors qu'il luy estoit inutile de penser jouer un autre personnage que le sien, dit que Floriandre estoit mort sans doute, mais qu'il ne sçavoit pourquoy elle croyoit qu'il s'appellast aussi Floriandre ». Ibid., tome III, p.89.
} 
il avoit falsifié ses affections, et corrompu l'amour qui est le plus doux lien de la société des hommes. ${ }^{97}$

Ainsi se met en place un réseau de démystification entre les personnages des romans, procédé que l'on pourrait appeler de démystification interne, par lequel on se dispense de l'intervention d'un narrateur omniscient ou de l'auteur en personne.

Force nous est de constater, alors, une double vision du langage chez Sorel, l'un horstexte, l'autre spécifique au Francion et compensant la déception suscitée par le premier. Dans le livre, le langage devient le dernier repère de vérité sur les individus. Il est l'indice le plus sûr de l'identité : Hortensius a beau se travestir, son langage le trahit, et muni d'une épée et de bottes, il «n'avoit plus rien de Pedantesque que les discours ${ }^{98} »$. Francion mal vêtu a bien failli être accusé d'un vol, « si par certaines paroles et actions (il) n'eus(t) contraint chacun aussitost d'avoir une très bonne opinion de (lui) ${ }^{9} \gg$. Les deux exemples sont frappants et confirment notre conviction d'un anti-carnavalesque foncier chez Sorel : le travestissement imposé à l'ordre du monde finit toujours par être démasqué, par être considéré comme une pure fiction, grâce à un élément témoin et conservateur d'un ordo inaltérable. Ici encore, c'est toujours le fantasme d'une adéquation invincible entre le Vrai et le Verbe qui est à l'œuvre. D'ailleurs la démystification des mensonges concédés aux émetteurs langagiers est révélatrice. Francion écrit des sonnets à Diane, un autre lui en vole la paternité, mais finalement l'ordre se rétablit et le cambrioleur verbal est « recogneu pour un lourdaut ${ }^{100} »$. Aventure similaire pour Hortensius qui emprunte des vers à un auteur, et se voit dénoncé par une amie de Fremonde ; ou encore pour Francion, qui écrit de la part de Clérante à Luce, et qui est personnellement récompensé par la clairvoyante correspondante.

Ce même ordre du monde est défendu par Francion lui-même, comme le remarque Jean Serroy ${ }^{101}$, et le personnage est héros à double titre : d'abord comme figure centrale de l'œuvre, puis comme porteur des valeurs positives. Le rôle du genre comique est bien là, par son apologie de la vérité, et d'une organisation du monde qu'il importe avant tout de respecter. La satire ne vise donc pas une catégorie sociale plus ou moins qu'une autre, toutes sont concernées dans la mesure d'un équilibre de cet ordo que l'on défend. La préface de Francion de 1626 Aux Grands est claire à cet égard : "Celuy qui est paysan et qui vit fort bien en paysan, me semble plus loüable que celuy qui est nay Gentilhomme et n'en faict pas les actions $^{102} »$. Sorel, parti du constat initial du mensonge dans le monde, était finalement confiant en cette croyance dans la souveraineté de la vérité dont il dit être l'artisan. La scène symbolique du géant vicieux, qui tente d'anéantir les révélations de ses débordements, est représentative de cette croyance en une invincibilité de la vérité et de ces moyens de divulgation. Le géant a tout pouvoir, on s'en doute, sur le vieillard, peut-être est-ce même lui qui a placé un cadenas sur sa bouche, mais il ne pourra rien contre les langues qui caquetteront encore une fois coupées, ni contre le rocher qui ne sera pas même « seulement

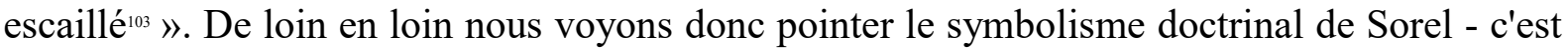
tout le discours de la Vérité invincible, et de la parole censée la véhiculer.

\footnotetext{
97 Ibid., tome IV, p.121.

98 Ibid., tome II, p.38.

99 Ibid., tome II, p.66.

100 Ibid., tome II, p.109.

101 «C'est contre ce monde à l'envers, où la valeur vénale tend à devenir la valeur morale, que le gentilhomme, porteur des traditions de son sang, part en guerre ». Jean Serroy, « Francion et l'argent ou l'Immoraliste et les Faux-Monnayeurs », XVIIème siècle, n¹05, 1974, p.6.

102 Francion, tome I p.X.

103 Ibid., tome I, p.136.
} 
C'est donc sur un constat optimiste que Sorel considère finalement sa parole romanesque, une parole qui accuse et conspue. En ce sens, comique et satirique étaient très proches. Mais que restait-il à dire après avoir raillé les pédants, les faux nobles, les avares, les poètes ou les paysans ? Comme l'a noté Philippe Salazar, la satire est « un genre éminemment performatif qui, sa mission achevée, doit apprendre à rentrer dans le silence $»^{104}$. Il restait à Sorel à rentrer dans le silence, ou bien à explorer d'autres voies littéraires. C'est ce dernier parti qu'il prendra, s'adonnant ensuite au traité chrétien ou scientifique, et, en une phase ultime, à la critique. Mais là encore, malgré toutes les méfiances qu'il avait senties et exprimées à propos du langage littéraire, en achevant sa production livresque par un exposé De la connoissance des Bons Livres, Sorel offrait à ses futurs exégètes un geste d'attachement à la littérature hautement symbolique.

Emmanuel Desiles Aix-Marseille Université

\footnotetext{
${ }^{104}$ Philippe-Joseph Salazar, « La satire, critique de l’éloquence », littératures classiques, n²4, 1995, p.182.
} 